\title{
Genetic Risk of Tuberculosis is Spread within the Hallmarks of the Disease
}

\author{
Jurica Vrbanec ${ }^{1}$, Petra Lederer-Dembic ${ }^{1}$, Ljiljana Bulat-Kardum ${ }^{1,2}$, Sanja Balen ${ }^{3}$, Randi Krog Eftedal ${ }^{1}$ and Zlatko Dembic ${ }^{{ }^{*}}$ \\ ${ }^{1}$ Molecular Genetics Laboratory, Department of Oral Biology, Dental faculty, University of Oslo, Norway \\ ${ }^{2}$ Section of Pulmology, Clinic for Internal Medicine, Clinical Hospital Center Rijeka, School of Medicine, University of Rijeka, Croatia \\ ${ }^{3}$ Clinical Institute for Transfusion Medicine, Clinical Hospital Center Rijeka, School of Medicine, University of Rijeka, Croatia
}

"Corresponding author: Zlatko Dembic, Molecular Genetics Laboratory, Department of Oral Biology, Dental faculty, University of Oslo, Norway, Tel: 4722840330 , Fax: 47228 40302; E-mail: zlatkod@odont.uio.no

Received date: April 22, 2016, Accepted date: May 14, 2016, Published date: May 19, 2016

Copyright: $\odot 2016$ Vrbanec J, et al. This is an open-access article distributed under the terms of the Creative Commons Attribution License, which permits unrestricted use, distribution, and reproduction in any medium, provided the original author and source are credited.

\begin{abstract}
Regarding genetic predisposition to tuberculosis, we suggest that the maximal risk for clinical manifestation requires complementation of sub-risks divided among the hallmarks of the disease. Clinical tuberculosis would only be revealed if at least one from each group of the genes encoding putative 5 (perhaps 7 ) hallmarks of the disease are mutated or changed epigenetically. These mutations/changes could be either sporadic (usually by the influence of the environment like other infection [HIV], nutrition, smoking, radiation etc.) or inherited. Avoidance of the immune attack is one of the hallmarks for TB that is shared with cancer. Perhaps, a similar immunotherapy as the recent one used in treating immunogenic types of cancer (anti-PD1, or/and anti-CTLA4) could be also successful in therapy of (multi-drug) resistant TB.
\end{abstract}

\section{Introduction}

Here we comment our recent findings regarding genetic risk for tuberculosis. In particular we refer to two kinds of studies: (A) the first stems from a collaborative effort in genome-wide screening (GWS) for genetic susceptibility to pulmonary tuberculosis in several ethnic groups of Caucasians [1], and the other (B) is from our educated guess studies [2-5].

A) The former study [1] identified a susceptibility locus on chromosome 6 in the human genome that is otherwise central in adaptive immunity. Namely, the association with disease was found in the locus encoding the HLA class II molecules. The products of $H L A$ genes are essential for $\mathrm{TB}$ antigen presentation on cells like macrophages and dendritic cells. During the type 1 immune responses particular HLA class II molecules in combination with TB antigenic peptide can cause CD4 T helper lymphocytes' activation and the secretion of interferon- $\gamma$ (IFN- $\gamma$ ), leading to pathogen-Mycobacterium tuberculosis clearance. Failure to do so would seem to present a high risk in contracting tuberculosis. Previous case-control studies found association with the disease of some HLA alleles in Cambodian (HLA$\mathrm{DQB1}^{*} 05: 03$, number of cases/controls $\left.[\mathrm{cs} / \mathrm{cn}]=126 / 88\right)$ [6] and Thai populations [7] (HLA-DQB1 ${ }^{*} 05: 01, \mathrm{cs} / \mathrm{cn}=82 / 160$ ), however those were not GWS. Two of us (L.B.K and Z.D.) contributed to recent GWS study that used 28 million markers spread across the genome and involved hundreds of thousands of individuals (9654 pulmonary tuberculosis patients and 294043 controls) [1]. The study found that three single nucleotide polymorphisms (SNPs) within the HLA class II loci were significantly associated with tuberculosis: rs557011 ( $\mathrm{p}<2 \times$ $\left.10^{-15}\right)$ and rs9271378 $\left(\mathrm{p}<3 \times 10^{-15}\right)$, both located between HLA-DQA1 and HLA-DRB1, and a missense variant encoding p.Ala210Thr in HLA-DQA1 ${ }^{*}$ 03:01 $\left(\mathrm{rs} 9272785, \mathrm{p}<1 \times 10^{-9}\right)$. Remarkably, the latter was not significantly associated with pulmonary TB in Croatians (cs/ $\mathrm{cn}=438 / 1008$ ), but it was significantly linked to increased risk for the disease in both, Icelandic population $\left(\mathrm{p}<3 \times 10^{-7}, \mathrm{cs} / \mathrm{cn}=3686 / 287427\right)$ and Russians $\left(\mathrm{p}<5 \times 10^{-4}, \mathrm{cs} / \mathrm{cn}=5530 / 5607\right)$. Recent case-control studies from other groups, despite using much lower number of individuals, corroborated the role of HLA class II in affecting the risk for TB in Ugandan (HLA DQB1 ${ }^{*} 03: 03, \mathrm{cs} / \mathrm{cn}=43 / 42$ ) [8] and Brasilian Amazon populations (HLA DRB1 ${ }^{*} 04, \mathrm{cs} / \mathrm{cn}=316 / 306$ ) [9]. Together, the data strongly suggest that poorly-Mycobacterium tuberculosisbinding HLA class II alleles might contribute to developing TB by being one of the many risks among multifactorial genetic hallmarks of tuberculosis.

Alongside the inappropriate HLA molecules (including DQA1 ${ }^{*}$ 03:01 found in our study [1]) that pose the greatest predisposition to tuberculosis in European Caucasians, additional molecules involved in the immune response to Mycobacterium tuberculosis bacterium from antigen presentation to effector cellular response might contribute to $\mathrm{TB}$ susceptibility and the search is on. The genome wide screenings, previously done in several other populations, identified other predisposing loci on chromosomes 15(q11-13) and X (The Gambia and South Africa) [10], 18q11 $\left(\mathrm{rs} 4331426, \mathrm{p}<6 \times 10^{-9}\right)$ (Ghana, The Gambia) [11], and 11p13 (rs2057178, $\mathrm{p}<2 \times 10^{-9}$ ) (Ghana, The Gambia, Indonesia, Russia) [12].

B) Perhaps, it could help to use an educated guess approach to look for additional risk factors. We did so by studying molecules important for the activation of antigen presenting cells like dendritic cells and macrophages. These molecules have roles in the host defense mechanisms against intracellular bacteria such as Mycobacterium tuberculosis, which live in macrophages. The activation process depends on molecules like Toll-like receptors (TLR) and proinflammatory cytokines including IFN- $\gamma$ and Interleukin (IL)-17. The latter stimulate macrophages to kill intracellular bacteria. Therefore, our educated guess studies involved genes encoding molecules such as IFN- $\gamma$, IL-17 (A and F), TLR-2 and TLR-10.

We have previously found association of particular IFNG polymorphisms with TB in our Caucasian subpopulation [3]. And, recently, we reported that genetic polymorphisms in genes coding for the anti-inflammatory TLR-10 protein could affect the risk for TB [2]. 
However, the association of SNPs in the pro-inflammatory IL17A and $I L 17 F$ genes (rs2275913 and rs763780, respectively) with tuberculosis, found previously in the Chinese population [13], could not be repeated in the Croatian Caucasians [2].

Upholding our finding that $I L 17 A$ polymorphism has no influence on TB risk in Caucasians came from recent meta-analysis that assessed rs22275913 polymorphism and concluded that neither Asians nor Caucasians have significant associations of this SNP with predisposition to $\mathrm{TB}$ [14]. Interestingly, significant association was observed in Asian population in another polymorphism in the IL17A gene rs3748067, which needs further assessment in Caucasians [14]. Furthermore, meta-analysis for $I L 17 F$ polymorphism rs763780 showed that, indeed, it might be linked to susceptibility to tuberculosis in Asians but not in Caucasians [14], confirming our previous report [2]. These results suggest that genetic TB risks regarding $I L 17$ genes might differ across various human subpopulations.

Another candidate gene, which has an important role in immunity against TB, is the Toll-like receptor 10 (TLR10), because it is an antiinflammatory factor [15]. TLR-10 chain associates with TLR-2 chain (a Mycobacterium tuberculosis-binding protein [16]) at the cell surface of macrophages. The TLR-10 / TLR-2 interaction is thought to diminish activation of macrophages (via IFN- $\gamma$ or IL17) to kill intracellular bacteria that would normally occur via heterodimeric complex made of TLR-2/TLR-1 (or TLR-2/TLR-6) in over $90 \%$ of healthy individuals. We reported previously that gene polymorphisms in TLR2 genes (that encode TLR-2 proteins either having a dominant-negative activity or disabled function) are associated with TB in Croatian population [17]. Molecular association with TLR10, which probably expunges TLR1 or TLR6 from the heterodimeric complex, can disable inflammatory action of the TLR2 protein. When we analyzed TLR10 SNP rs11096957 we found that it was associated with increased risk for TB, and, furthermore, homozygotes carrying the A allele had increased risk for developing the disease [2].

Why is there a different HLA class II allele found associated with tuberculosis in every tested subpopulation? And, why have some $I L-17(A / F)$ polymorphisms been found as risk factors in one human subpopulation but not in another?

An explanation could be that genome markers (polymorphisms in DNA that affect protein expression and function) are being changed by natural selection faster than anticipated, and thus they are not easily recognized for what they stand for. Alternatively, they represent pointers for a collection of malfunctioned biologic processes important for the defense against Mycobacterium tuberculosis bacillus that were distributed unequally in humans by migration and genetic drift. We favor the latter explanation. For example, in our recent study [1], we found a failure in the antigen presentation process (by not having the appropriate HLA molecule to present a Mycobacterium tuberculosis peptide to $\mathrm{T}$ cells) as a risk for contracting pulmonary $\mathrm{TB}$ Furthermore, another failed process might also pose a risk: CD4 T cells (in cell contact with macrophages having perhaps the "wrong" allele of TLR10) might secrete lower amounts of IFN- $\gamma$ needed to (activate the same macrophages and) destroy intracellular Mycobacterium tuberculosis [2]. We believe these studies do not need confirmations (that exactly the same allele or polymorphism affect TB risk in other human subpopulation), but rather complementation with genes belonging to the same putative hallmark of TB.

\section{The Hypothesis about Hallmarks of Tuberculosis}

We suggest that the complex genetic susceptibility to tuberculosis is theoretically comparable to risk of developing cancer. For cancer, in order to become a deadly disease, at least ten genes need to be mutated or epigenetically changed. These must belong to ten groups of biologic processes that are characteristics (hallmarks) of all cancers [18]. Every hallmark comprises a set of genes, whose mutated or epigenetically changed forms are often found assembled in a cancer cell with a different combination of other 9 hallmarks' oncogenic alleles, in each individual case. Moreover, many mutated or modified genes might be present in a cancer stem cell, but if they comprise all ten hallmarks, the risk for developing deadly form of cancer is maximized.

We suggest that a similar analysis can be exploited in explaining the risk for developing clinical tuberculosis. The ten groups of genes mutated in cancer are deliberately called "hallmarks" as they represent failed functional processes or pathological characteristics of the disease [18]. With the same aim let us define the hallmarks of tuberculosis, and then, in the next stage, plan to analyze whether tuberculosis has developed in individuals that carry a set of polymorphisms (either mutations or epigenetic malformations) belonging to all proposed hallmark groups (see below and Figure 1).

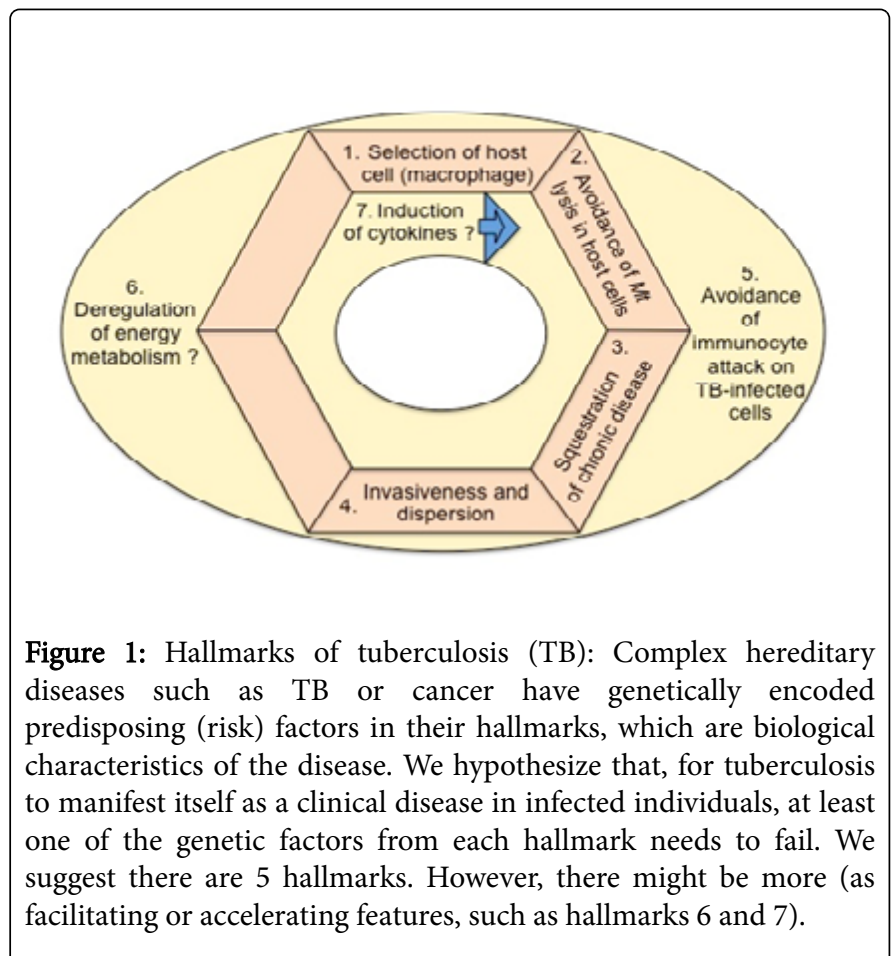

Up to $10 \%$ of human population have largely unknown defects in the immunity causing them to develop tuberculosis. Perhaps several hallmarks/groups of predisposing factors involving innate and adaptive immunity contribute to these defects. Their number is unknown but here we propose (at least) 5. The hallmarks of tuberculosis are (Figure 1):

1. Selection of the proper cellular host (i.e. pulmonary macrophage, including TLR2 binding),

2. Avoidance of the phagocytic capability of the cellular host (i.e. blocking phago-lysosome), 
3. Producing granulomatous (inflamed) physical barrier to sequester its replicative capability (perhaps, by producing toxins and molecules that dis-regulate the innate (and specific) immune responses, or recruit anti-inflammatory molecules to keep the balance with pro-inflammatory ones) and avoid resolution of inflammation making it chronic,

4. Enforcing a parasitic state in the host organism, leading to invasion of Mycobacterium tuberculosis into other tissues and organs (dispersion, i.e. milliary $\mathrm{TB}$ ), and producing infectious particles to make a spread by air.

5. Avoiding attack by the specialized immune cells (CD4 Th1 cells or NK cells that secrete IFN- $\gamma$ ), for example, by selecting a host with a "wrong or faint signature" (i.e. a hypomorphic HLA class II, "bad" peptide transporter gene, low IL-1 activity that decreases expression of class II, "bad" vitamin D receptor, etc.) for the activation of effector T cells or NK cells. This hallmark might be a facilitating one, i.e. it could affect other 4 hallmarks.

We propose that, in order for tuberculosis to manifest itself as a clinical disease, at least one of the molecular factors from each hallmark need to fail ( 5 in total). This can happen by chance, for example, when a child inherits a predisposing line-up of polymorphisms or epigenetic changes in genes encoding such factors. A single hallmark change cannot cause the disease alone. However, it can contribute to spreading the risk across the globe with human migration. For example, the clinical disease (in its worst form) would only ensue if a host has a genetic setup made of the following genes whose polymorphic alleles are hypothetically spread among the abovementioned 5 groups:

1. A TLR2 variant that preferentially associates with TLR1 or TLR6 and binds Mycobacterium tuberculosis TLR2 variants that do not bind Mycobacterium tuberculosis would render such hosts unable to be infected, and perhaps that is why some persons that died by accidental death and no clinical disease had Mycobacterium tuberculosis in lungs, but outside macrophages [19].

2. A variant encoding a relatively "weak enzyme" (or process) that cannot sufficiently digest the thick Mycobacterium tuberculosis capsule.

3. A variant coding for a putative "bad molecule", possibly a cytokine or its receptor, or an intracellular signaling molecule that can inhibit the activation of macrophages, which normally occurs via IFN- $\gamma$ or IL-17, after the contact with the Mycobacterium tuberculosis bacterium. (This "bad molecule" includes the latter two and possibly other cytokines belonging to the IL-12 / IFN- $\gamma$ axis of macrophage activation).

4. A variant encoding a putative "anti-inflammatory" molecule (cytokine, receptor or soluble signaling molecule) that would allow "leaking" of the Mycobacterium tuberculosis into the circulation, thereby spreading the infection to other tissues. (A putative TLR10 variant could preferentially bind TLR2, diminish inflammatory response, and thus allow for such leakage. Alternatively, a variant of a functional TLR2 chain that mostly gives low inflammatory signal either as a heterodimer with TLR1 or TLR6, or in the ability to bind preferentially TLR10 rather than TLR1 or TLR6. However, other molecules involved in the inhibition of innate immunity might also be involved.)

5. A variant encoding a protein molecule that would help Mycobacterium tuberculosis to avoid attack by the immune cells on the infected cells like macrophages, giant cells and cells in other tissues. This hallmark includes "bad recognition" of Mycobacterium tuberculosis peptides by $\mathrm{T}$ cells (for example, inappropriate HLA) during lymphocytes 'priming and targeting of their effector cells. Alternatively, in those individuals that have appropriate HLA molecules, perhaps a "bad" variant of a gene encoding a cytokine involved in the activation of CD4 and CD8 T cells might be present, allowing Mycobacterium tuberculosis to squeeze through the defense of the adaptive immune system. Additional alternatives could include variants that incapacitate NK cells, activate Tregs (that, in turn, inhibit antiMycobacterium tuberculosis response), or perhaps inhibit the immune system via other known mechanism. For these alternatives, most of the literature was cited in the manuscripts that we comment here $[1,2]$.

We further suggest that defective risk factors (coming from all 5 hallmarks) are assembled differently in various human subpopulations. Perhaps, that is why there is a population-specific HLA Class II molecule that was found associated with tuberculosis, which probably poorly presents Mycobacterium tuberculosis peptides. Along the same lines we could explain differences between Chinese and Caucasian populations found in association with tuberculosis of the $\operatorname{IL17}(A / F)$ genes' SNPs. Perhaps due to overlapping functionality of some cytokines there might be an IL17-like-cytokine failure to complement putative hallmark no. 3 of TB risk in Caucasians.

How can we explain an increased risk for tuberculosis in patients with so-called "Mendelian susceptibility to Bacille Calmette-Guerin (BCG)" or after HIV infection? In both cases namely the risk seems to depend on a single trait, either a dominant gene defect in the IL-12/ IFN- $\gamma$ axis (i.e. IL-12 receptor, IFN- $\gamma$ receptor) or in the depletion of CD4 T cells, respectively.

For the first problem, BCG is a live, attenuated preparation of vaccine that imitates Mycobacterium tuberculosis. Despite having much milder form of reaction in normal population, BCG can cause death in children that for hereditary reasons lack expression of, for example, the IFNGR1 gene [20]. Similar reports, which are very rare cases, have been documented for other genes in the IL-12 and IFN- $\gamma$ signaling pathways (for a review see [21]). It seems that a sole hallmark-3 factor (bad IFNGR1 allele) can cause BCG-itis and perhaps tuberculosis [20]. We explain this by postulating that predisposing factors from other hallmarks (groups 1, 2, 4, and 5) must have been present already in such cases. Let's analyze which: TLR2 should be there (hallmark 1), the "bad enzyme" (hallmark 2), and the "bad allele" encoding IL-17 or TLR10 (hallmark 4) might also be present, and lastly, CD4 T cells (hallmark 5) could not be fully functional without the receptor (IFNGR1) that is essential for their effector ability(s). Perhaps hallmark 4 (spreading to other organs) might not be present, as BCG (in this particular rare case) could cause death differently (i.e. insufficiency of a single organ) from Mycobacterium tuberculosis. Furthermore, let us assume that the hallmark 5 defines risk of TB as a consequence of (at worst) a hypomorphic IFNGR1 and not a complete "knockout-type" allele. In the latter case, both hallmarks (3 and 5) would have failed. Therefore, the rare cases of Mendelian predisposition to BCG are individuals whose lack of hallmarks 3 and 5 (and the event of BCG vaccination) has brought to light this disease. Perhaps there are some who do not have risk affecting hallmarks 1, 2 or 4 and, according to our prediction, should not have such illness, but were not detected, simply because they were not ill and thus went unrecognized (yet). Only total genome DNA sequencing of a larger 
proportion of humans (>million) would eventually reveal (or not) this issue.

Regarding the second issue (an HIV infection), the hallmark-5 factor is absent due to HIV-mediated depletion of functional CD4 T cells (or dendritic cells expressing CD4). If infected with Mycobacterium tuberculosis, development of clinical tuberculosis is seen in majority of such patients. This several fold increase in TB risk (up from $5-10 \%$ seen in normal population) we explain by postulating that other 4 hallmarks have been compromised in HIV-patients clinically ill with TB. Here, we need more data to support this conjecture. Theoretically, helper CD4 Th1 cells secrete IFN- $\gamma$, which is also the hallmark no. 3. Similarly to previous argument with Mendelian susceptibility to BCG, here the risk for TB would already include defects in hallmarks 3 and 5.

Furthermore, the fact that not all (of HIV infected and in contact with Mycobacterium tuberculosis) become ill with TB might mean that some (if not all) risk factors have protective alleles. For the latter, protective candidates should be found among the remaining hallmarks (1,2 and 4), possibly acting in concert with each other. In support, we already reported some evidence suggesting that individuals who are high secretors of IFN- $\gamma$ might be protected against TB in normal (HIV-negative) population [3].

Perhaps we can increase the number of listed hallmarks (Figure 1) by dividing some or adding new ones, should there be good arguments and evidence for it. It is reasonable to assume that new knowledge would increase the number of TB hallmarks in the future. If we draw a parallel with cancer, again, then certain facilitating and/or accelerating hallmarks are missing from the above list. For example, is it possible that the growing Mycobacterium tuberculosis within the macrophage might affect metabolism of fat, amino acids or carbohydrates (Hallmark no. 6 in Figure 1)? In addition, and as a speculative question, can Mycobacterium tuberculosis provoke secretion of some hormones or locally produced cytokines, which would, in turn, accelerate the establishment of other hallmarks of tuberculosis (Hallmark no. 7 in Figure 1)?

Consequently, we believe that it is important to study genetic risk factors for TB in every human subpopulation similarly as it is done for cancer, especially now that novel immunotherapies, have opened new ways to treatment of advanced cases.

In particular, nivolumab (pembrolizumab or pidilizumab; anti-PD1) and ipilimumab (anti-CTLA4) could be tried as they counter the hallmark that is supposedly shared with cancer- namely, avoidance of the immune attack. These immunotherapies have already shown their clinical relevance in treatment of metastasized melanoma or other immunogenic carcinomas. Perhaps, by better defining hallmarks of tuberculosis we could open possibilities for novel anti-TB therapies, besides the well-known antibiotic regimens, some that can help in fight against the resistant and multi-resistant Mycobacterium tuberculosis types.

\section{References}

1. Sveinbjornsson G, Gudbjartsson DF, Halldorsson BV (2016) HLA class II sequence variants influence tuberculosis risk in populations of European ancestry. Nat Genet 48: 318-322.

2. Bulat-Kardum LJ, Etokebe GE, Lederer P, Balen S, et al. (2015) Genetic Polymorphisms in the Toll-like Receptor 10, Interleukin (IL)17A and IL17F Genes Differently Affect the Risk for Tuberculosis in Croatian Population. Scand J Immunol 82: 63-69.
3. Etokebe GE, Bulat-Kardum L, Johansen MS, Knezevic J, Balen S, et al. (2006) Interferon-gamma gene (T874A and G2109A) polymorphisms are associated with microscopy-positive tuberculosis. Scand J Immunol 63: 136-141.

4. Bulat-Kardum L, Etokebe GE, Knezevic J, Balen S, Matakovic-Mileusnic $\mathrm{N}$, et al. (2006) Interferon-gamma receptor-1 gene promoter polymorphisms (G-611A; T-56C) and susceptibility to tuberculosis. Scand J Immunol 63: 142-150.

5. Fraser DA, Bulat-Kardum L, Knezevic J, Babarovic P, MatakovicMileusnic N, et al. (2003) Interferon-gamma receptor-1 gene polymorphism in tuberculosis patients from Croatia. Scand J Immunol 57: 480-484.

6. Goldfeld AE, Delgado JC, Thim S, Bozon MV, Uglialoro AM, et al. (1998) Association of an HLA-DQ allele with clinical tuberculosis. JAMA 279: 226-228.

7. Vejbaesya S, Chierakul N, Luangtrakool K, Srinak D, Stephens HA (2002) Associations of HLA class II alleles with pulmonary tuberculosis in Thais. Eur J Immunogenet 29: 431-434.

8. Wamala D, Buteme HK, Kirimunda S, Kallenius G, Joloba M (2016) Association between human leukocyte antigen class II and pulmonary tuberculosis due to mycobacterium tuberculosis in Uganda. BMC Infect Dis 16: 23.

9. Souza de Lima D, Morishi Ogusku M, Porto Dos Santos M, de Melo Silva CM, Alves de Almeida V, et al. (2016) Alleles of HLA-DRB1*04 Associated with Pulmonary Tuberculosis in Amazon Brazilian Population. PLoS One 11: e0147543.

10. Bellamy R, Beyers N, McAdam KP, Ruwende C, Gie R, et al. (2000) Genetic susceptibility to tuberculosis in Africans: a genome-wide scan. Proc Natl Acad Sci U S A 97: 8005-8009.

11. Thye T, Vannberg FO, Wong SH, Owusu-Dabo E, Osei I, et al. (2010) Genome-wide association analyses identifies a susceptibility locus for tuberculosis on chromosome 18q11.2. Nat Genet 42: 739-741.

12. Thye T, Owusu-Dabo E, Vannberg FO, van Crevel R, Curtis J, et al. (2012) Common variants at $11 \mathrm{p} 13$ are associated with susceptibility to tuberculosis. Nat Genet 44: 257-259.

13. Peng R, Yue J, Han M, Zhao Y, Liu L, et al. (2013) The IL-17F sequence variant is associated with susceptibility to tuberculosis. Gene 515: 229-232.

14. Zhao J, Wen C, Li M (2016) Association Analysis of Interleukin-17 Gene Polymorphisms with the Risk Susceptibility to Tuberculosis. Lung 194: 459-467.

15. Oosting M, Cheng SC, Bolscher JM, Vestering-Stenger R, Plantinga TS, et al. (2014) Human TLR10 is an anti-inflammatory pattern-recognition receptor. Proc Natl Acad Sci U S A 111: E4478-4484.

16. Underhill DM, Ozinsky A, Smith KD, Aderem A (1999) Toll-like receptor-2 mediates mycobacteria-induced proinflammatory signaling in macrophages. Proc Natl Acad Sci U S A 96: 14459-14463.

17. Etokebe GE, Skjeldal F, Nilsen N, Rodionov D, Knezevic J, et al. (2010) Toll-like receptor $2(\mathrm{P} 631 \mathrm{H})$ mutant impairs membrane internalization and is a dominant negative allele. Scand J Immunol 71: 369-381.

18. Hanahan D, Weinberg RA (2011) Hallmarks of cancer: the next generation. Cell 144: 646-674.

19. Hernandez-Pando R, Jeyanathan M, Mengistu G, Aguilar D, Orozco H, et al. (2000) Persistence of DNA from Mycobacterium tuberculosis in superficially normal lung tissue during latent infection. Lancet 356: 2133-2138.

20. Altare F, Jouanguy E, Lamhamedi-Cherradi S, Fondaneche MC, Fizame C, et al. (1998) A causative relationship between mutant IFNgR1 alleles and impaired cellular response to IFNgamma in a compound heterozygous child. Am J Hum Genet 62: 723-726.

21. Casanova JL, Abel L (2002) Genetic dissection of immunity to mycobacteria: the human model. Annu Rev Immunol 20: 581-620. 
Citation: Vrbanec J, Lederer-Dembic P, Bulat-Kardum L, Balen S, Eftedal RK, et al. (2016) Genetic Risk of Tuberculosis is Spread within the Hallmarks of the Disease. Immunother Open Acc 2: 117. doi:10.4172/2471-9552.1000117 